AIP/123-QED

\title{
Revisiting pyramid compression to quantify flexoelectricity: a 3D simulation study
}

\author{
Amir Abdollahi, Daniel Millán, Christian Peco, Marino Arroyo, and Irene Arias* \\ Laboratori de Càlcul Numèric (LaCàN), \\ Universitat Politècnica de Catalunya (UPC), \\ Campus Nord UPC-C2, E-08034 Barcelona, Spain. ${ }^{\dagger}$
}

(Dated: October 19, 2015)

\begin{abstract}
Flexoelectricity is a universal property of all dielectrics, by which they generate a voltage in response to an inhomogeneous deformation. One of the controversial issues in this field concerns the magnitude of flexoelectric coefficients measured experimentally, which greatly exceed theoretical estimates. Furthermore, there is a broad scatter amongst experimental measurements. The truncated pyramid compression method is one of the common setups to quantify flexoelectricity, the interpretation of which relies on simplified analytical equations to estimate strain gradients. However, the deformation fields in 3D pyramid configurations are highly complex, particularly around its edges. In the present work, using three-dimensional self-consistent simulations of flexoelectricity, we show that the simplified analytical estimations of strain gradients in compressed pyramids significantly overestimate flexoelectric coefficients, thus providing a possible explanation to reconcile different estimates. In fact, the interpretation of pyramid compression experiments is highly nontrivial. We systematically characterize the magnitude of this overestimation, of over one order of magnitude, as a function of the truncated pyramid configuration. These results are important to properly characterize flexoelectricity, and provide design guidelines for effective electromechanical transducers exploiting flexoelectricity.

PACS numbers: 77.65.-j, 77.80.bn, 77.90.+k
\end{abstract}




\section{INTRODUCTION}

Flexoelectricity is a two-way electromechanical coupling mechanism between electric polarization and strain gradients or strain and polarization gradients, rather than between polarization and strain as in piezoelectricity ${ }^{1,2}$. This material property may enable electromechanical transducers made out of non-piezoelectric materials ${ }^{3-6}$, or new ways to address information in ferroelectric thin films ${ }^{7}$. Because strain gradients disrupt in general the inversion symmetry of the microscopic structure of a material, flexoelectricity is very general for diclectrics, unlike piezoclectricity, only present in non-centrosymmetric materials. On the other hand, flexoelectricity is strongly size-dependent because strain gradients are inversely proportional to structural size. Therefore, despite its universality, the flexoelectric effect is significant only at micro- and nanoscopic scales, making its experimental characterization challenging.
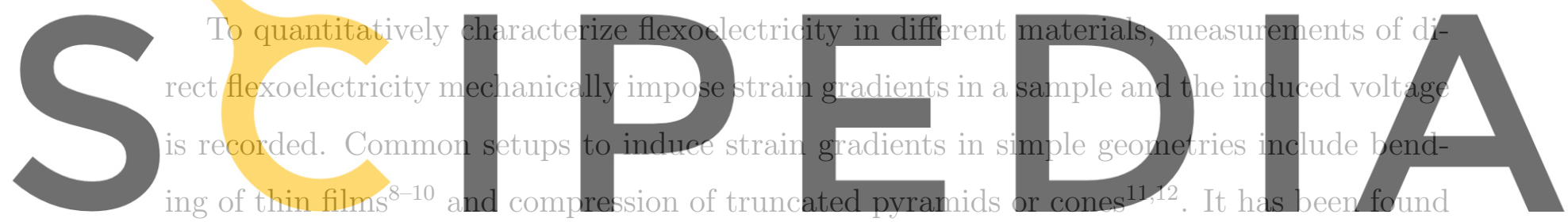

that flexoelectric coefficients, are particularly large in materials with high dielectric conRegister for free at https//www.scipedia.com to download the version without the watermark stants, such as ferroelectrics $9,11,13-15$. However, the magnitude of these coefficients greatly

exceeds theoretical estimates ${ }^{1,16,17}$. This discrepancy has lead to a controversy in the fieid of flexoelectricity, demanding further investigations into the quantification of the flexoelectric response $^{18}$. While recent developments in the field demonstrate that the surface contributions to the flexoelectric response may be a possible explanation ${ }^{19-21}$, there is still a limited quantitative understanding on the flexoelectric coefficients. To translate an experimental measurement into a material parameter estimation, one must resort to a model. Here, we examine if the modeling assumptions underlying previous experimental estimations of flexoelectricity may provide a source of significant error.

The most widely used model for flexoelectricity is the linear continuum theory of flexoelectricity (see the recent reviews ${ }^{18,22,23}$ ). In this theory, flexoelectricity is represented by a fourth-order flexoelectric coupling tensor, whose symmetry is well understood ${ }^{24-26}$. Mathematically, the self-consistent electro-mechanical field equations of flexoelectricity are a coupled system of 4th order partial differential equations (PDEs). Despite analytical solu- 
tions are starting to emerge for simple geometries and loads ${ }^{27,28}$, most of the field operates with approximate solutions valid under very restrictive assumptions ${ }^{11,29,30}$. Furthermore, to interpret experiments, the two-way flexoelectric coupling is often ignored, by estimating strain gradients from elasticity alone ${ }^{10}$.

To go beyond these simple approximate solutions, on can resort to computational methods, but because the equations involve high-order spatial derivatives, flexible methods such as conventional finite elements cannot be used. On rectangular or brick geometries, finite difference calculations have been applied to flexoelectricity ${ }^{31-33}$. To deal with more general geometries with non-uniform grid refinement, we have recently resorted to meshfree methods, relying on smooth basis functions ${ }^{34}$, to solve numerically the continuum equations of flexoelectricity in 2D ${ }^{35}$. Surprisingly, we found that previous simplified calculations on beam and truncated triangle configurations provided only rough order-of-magnitude estimations of the flexoelectric response. These previous results suggest that in the 3D truncated pyramid

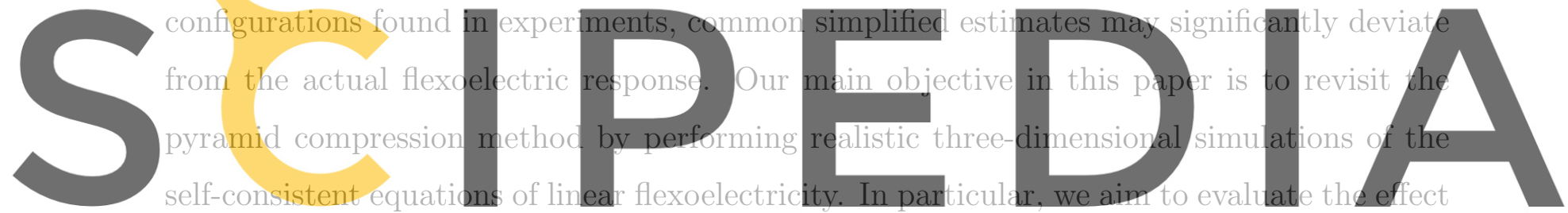

of pyramid geometry and boundary conditions on the flexoelectric response. Register for free at https//www.scipedia.com to download the version without the watermark

The structure of the paper is as follows. A summary of the continuum theory of flexo-

electricity along with the computational model is presented in Section II. We then revisit the truncated pyramid experiment through 3D simulations in Section III and, in particular, discuss the sources of overestimation in the flexoelectric response. A systematic study of this response as a function of the truncated pyramid configuration are presented in Section IV. The last section is the conclusion.

\section{THEORY, COMPUTATIONAL MODEL, AND MATERIAL PARAMETERS}

The constitutive equation for the electric polarization $\mathbf{P}$ in a linear dielectric solid possessing flexoelectricity is ${ }^{1}$ :

$$
P_{i}=\chi_{i j} E_{j}+\mu_{k l i j} \nabla_{j} \varepsilon_{k l}
$$

where $\mathbf{E}$ is the electric field and $\varepsilon$ is the mechanical strain. The dielectric response is described by the first term, where $\chi$ is the second-order dielectric susceptibility tensor. The 
flexoelectric effect is introduced by the second term, where $\mu$ is the fourth-order tensor of flexoelectricity and $\nabla \varepsilon$ is the strain gradient. Given the electric polarization in Eq. (1), the electric displacement $\mathbf{D}$ is obtained as

$$
D_{i}=\varepsilon_{0} E_{i}+P_{i}=\kappa_{i j} E_{j}+\mu_{k l i j} \nabla_{j} \varepsilon_{k l},
$$

where $\varepsilon_{0}$ is the permittivity of free space and $\kappa_{i j}$ is the second-order dielectric tensor. Equation (2) determines the electrostatic state of the solid, where the electric field derives from the electric potential $\phi, E_{i}=-\phi_{, i}$.

The thermodynamically conjugated effect to the direct flexoelectric response in $\mathrm{Eq}$. (2) is the converse flexoelectric effect, introduced via the constitutive equation for the mechanical

$$
\sigma_{i j}=\mathbb{C}_{i j k l} \varepsilon_{k l}+\mu_{l i j k} E_{l, k}-h_{i j k l m n} \varepsilon_{l m, n k},
$$

where the first term is the elastic response, $\mathbb{C}$ being the fourth-order tensor of elastic moduli.

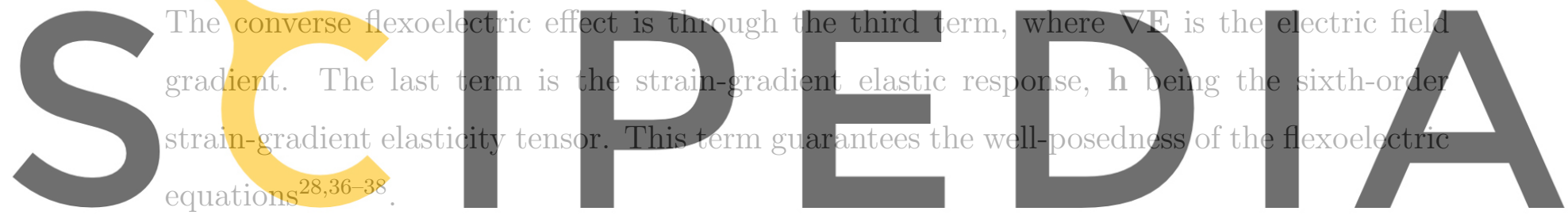

Equations (2) and (3) can be shown to derive from an electro-mechanical enthalpy energy Register for free at https//www.scipedia.com to download the version without the watermark density for the linear flexoelectric solid

$$
\begin{gathered}
\mathcal{H}\left(\varepsilon_{i j}, E_{i}, \varepsilon_{j k, l}\right)=\frac{1}{2} \mathbb{C}_{i j k l} \varepsilon_{i j} \varepsilon_{k l}-\mu_{i j k l} E_{i} \varepsilon_{j k, l}-\frac{1}{2} \kappa_{i j} E_{i} E_{j} \\
+\frac{1}{2} h_{i j k l m n} \varepsilon_{i j, k} \varepsilon_{l m, n}
\end{gathered}
$$

as

$$
\hat{\sigma}_{i j}=\frac{\partial \mathcal{H}}{\partial \varepsilon_{i j}}, \quad \widetilde{\sigma}_{i j k}=\frac{\partial \mathcal{H}}{\partial \varepsilon_{i j, k}}, \quad \hat{D}_{i}=-\frac{\partial \mathcal{H}}{\partial E_{i}},
$$

where the mechanical stress is obtained from the usual contribution $\hat{\sigma}$ and the higher-order (hyper) stress $\widetilde{\sigma}$ as

$$
\sigma_{i j}=\hat{\sigma}_{i j}-\widetilde{\sigma}_{i j k, k}
$$

The total enthalpy of the system results from integrating $\mathcal{H}$ over the domain, and accounting for the contributions of the external loadings. We refer to Ref. ${ }^{35}$ for a complete description of the theory and its Galerkin numerical discretization. For the reader's convenience, we provide in A details about the numerical implementation in 3D. We have carefully checked numerical convergence, as shown in Fig. 2. 

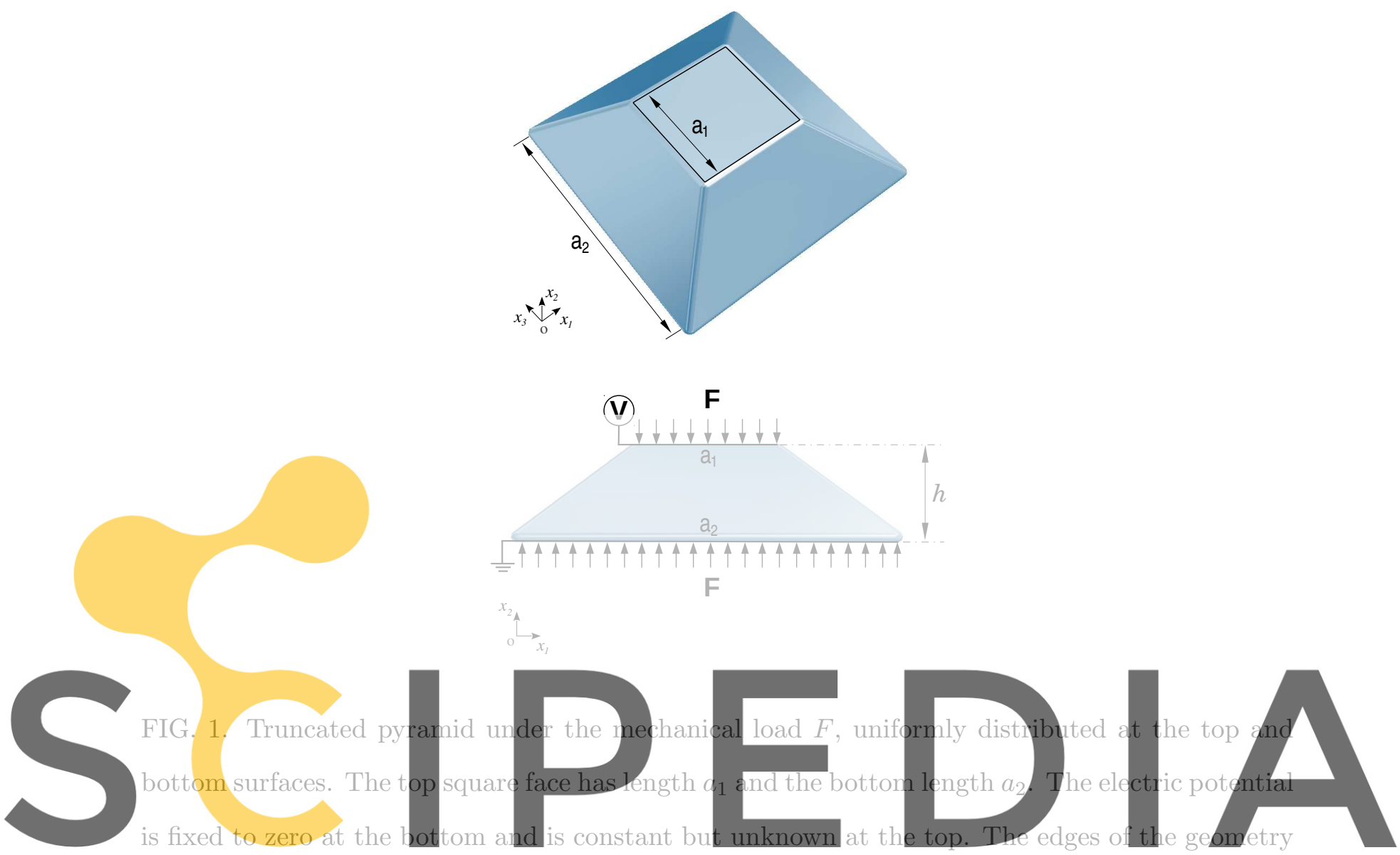

Register for free at https//www.scipedia.com to download the version without the watermark

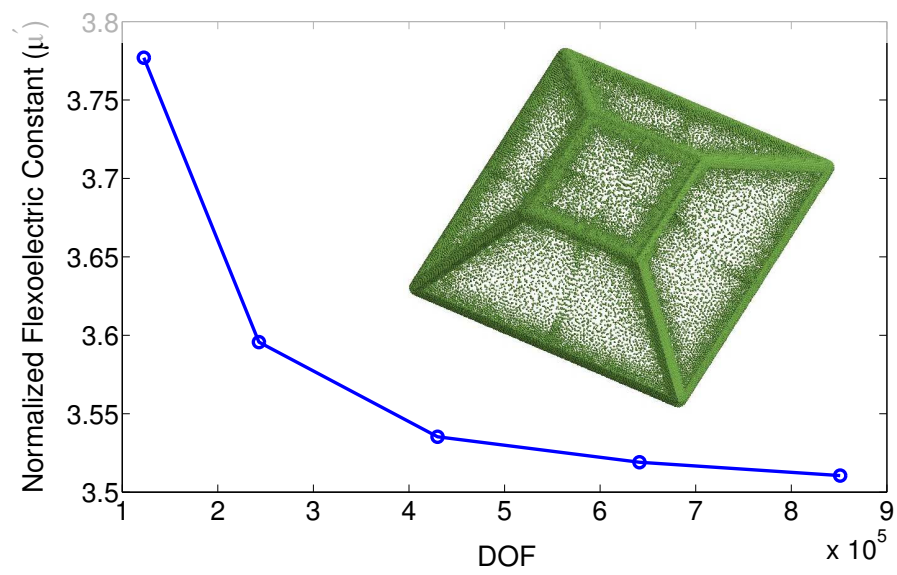

FIG. 2. Convergence study of the numerical simulations. Normalized flexoelectric constant $\mu^{\prime}$ as a function of the number of degrees of freedom (DOF), i.e. the number of nodes times 4 (3 components of the displacement field and the electric potential). The inset shows a representative computational node set. 
To perform numerical simulations, we consider truncated pyramid geometries as shown in Fig. 1. A total force of magnitude $F$ is applied uniformly at the top and bottom square faces, whose lateral dimensions are $a_{1}$ and $a_{2}$. The electric potential is fixed to zero at the bottom and to a constant but a priori unknown value $V$ at the bottom electrode, which is found as a result of the numerical calculation.

The material constants are chosen to fit the behavior of a strongly flexoelectric material, Barium Strontium Titanate (BST) in its non-piezoelectric (paraelectric) phase at room temperature. We adopt a simple choice for the symmetry of the material tensors, but general enough to capture the multi-dimensional couplings of the field equations. We consider isotropic elasticity and permittivity and adopt cubic symmetry for the flexoelectric tensor. Therefore, there are only two independent elastic constants, the Young's modulus E and the Poisson's ratio $\nu$, one dielectric permittivity constant $\kappa_{11}$, two strain-gradient elasticity constants $h_{11111}$ and $h_{122122}$ (or in matrix notation $h_{11}$ and $h_{12}$, see A), and three independent

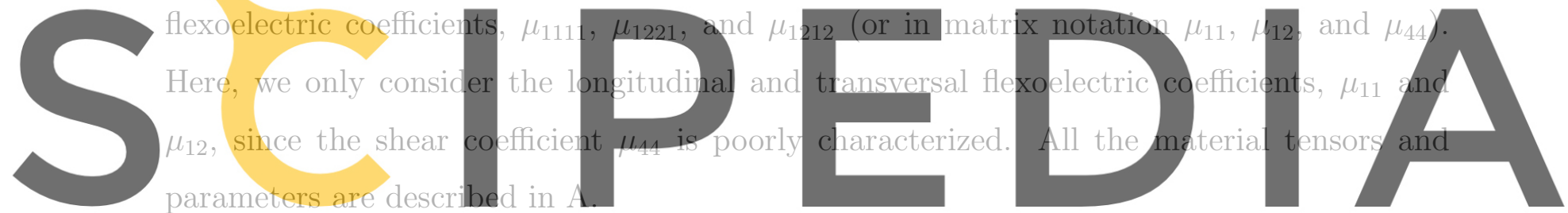

Register for free at https//www.scipedia.com to download the version without the watermark

III. REVISITING THE TRUNCATED PYRAMID EXPERIMENT THROUGH 3D SIMULATIONS

The applied force on the truncated pyramid generates different tractions at the top and bottom surfaces due to their different areas. As a result, a strain gradient and thus a flexoelectric polarization are generated. Thus, but adopting a pyramid geometry, a nonpiezoelectric solid behaves effectively as a piezoelectric solid. To quantify this effective piezoelectricity, one can consider a comparison piezoelectric solid with a parallelepiped geometry of square base of size $a_{2}$, height $h$, and piezoelectric coupling tensor e, which produces the same polarization as the flexoelectric solid with pyramid geometry. Let us assume that the material only has non-zero longitudinal flexoelectric coefficient $\mu_{11}$ and elastic constant $c_{11}=E(1-\nu) /(1+\nu)(1-2 \nu)$, and that the through-thickness longitudinal strain $\varepsilon_{22}$ is the only non-zero component. In the piezoelectric solid, strain is constant and given by $\bar{\varepsilon}_{22}=F /\left(c_{11} a_{2}^{2}\right)$. In the pyramid, by interpolating linearly the strain at top and bottom 
surfaces, the strain gradient can be estimated as $\varepsilon_{22,2}=\bar{\varepsilon}_{22}(R-1) / h$, where $R=\left(a_{2} / a_{1}\right)^{2}$ is the area ratio, see Fig. 1. Then, the equation equating the polarization in the flexoelectric pyramid and in the comparison piezoelectric solid

$$
e_{i j k} \varepsilon_{j k}=\mu_{k l i j} \nabla_{j} \varepsilon_{k l}
$$

simplifies to ${ }^{11}$

$$
e_{33}=\mu_{11}\left(\frac{R-1}{h}\right)
$$

providing a definition for the effective piezoelectric constant $e_{33}$ of the pyramid. Experimentally, if the electric potential difference between electrodes $V$ resulting from an applied force $F$ is recorded, $e_{33}$ can also be computed by dividing the nominal polarization in the sample

$-\chi_{11} V / h$ by the nominal strain as

$$
e_{33}=-\frac{\chi_{11} V}{h \bar{\varepsilon}_{22}}
$$
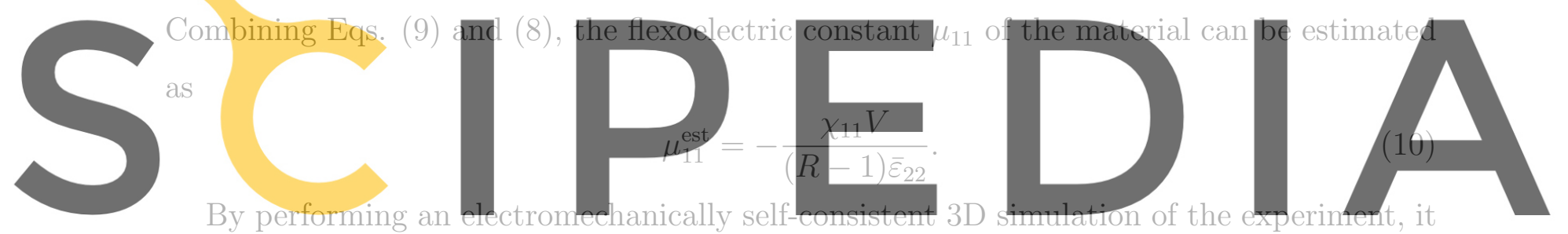

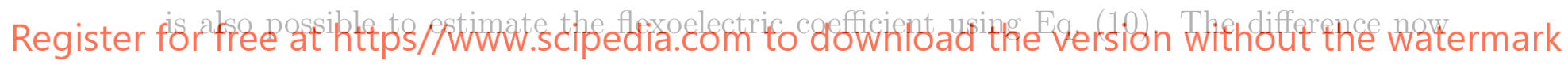

is that the actual flexoelectric coefficient $\mu_{11}$ used to produce the simulation is known, and

therefore we can define a normalized flexoelectric constant $\mu^{\prime}=\mu_{11}^{\text {est }} / \mu_{11}$, measuring the accuracy of the simple estimation used to interpret experiments. If the analytical formula in (10) is a good estimate of the flexoelectric response, the normalized flexoelectric constant $\mu^{\prime}$ should be close to one.

To examine this point, we perform a case study simulation inspired in the work of Ref. ${ }^{3}$, where experiments were performed on an array of truncated pyramids under compression. In these experiments, electrodes were deposited on the top and bottom sides of the pyramids to collect the induced charges. The dimensions of the pyramids in the experiments, also used here, are $a_{2}=2.72 \mathrm{~mm}, a_{1}=1.13 \mathrm{~mm}$, and $h=0.76 \mathrm{~mm}$. The 3 -D simulation of this pyramid, subjected to a load of $F=200 \mathrm{~N}$, predict an induced voltage of $V=-17.1 \mathrm{~V}$ on the top electrode, as shown in Fig. 3(a). With these data, a normalized flexoelectric constant $\mu^{\prime}=3.5$ is obtained, indicating more than a three-fold overestimation of the flexoelectric constant by the simple analytical estimate. 

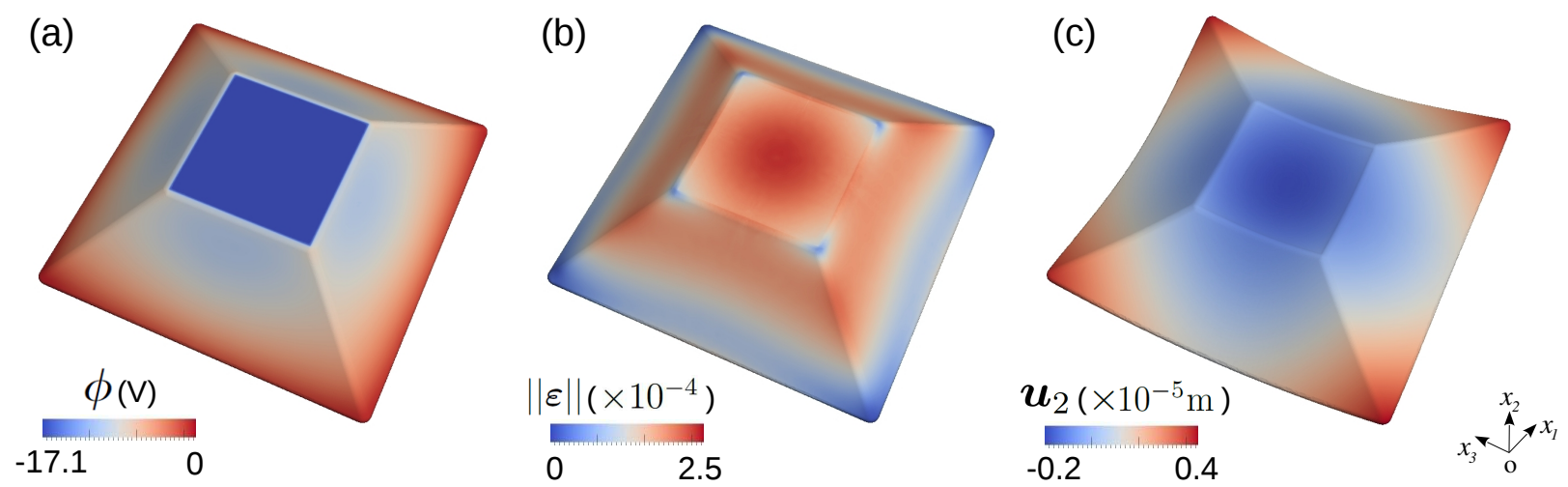

FIG. 3. Distribution of (a) the electric potential $\phi$ and (b) the strain norm $\|\varepsilon\|$ in the truncated pyramid under compression. (c) Distribution of the through-thickness mechanical displacement $u_{2}$ in the deformed configuration of the pyramid. The deformation is exaggerated by a factor of 10 for clarity.
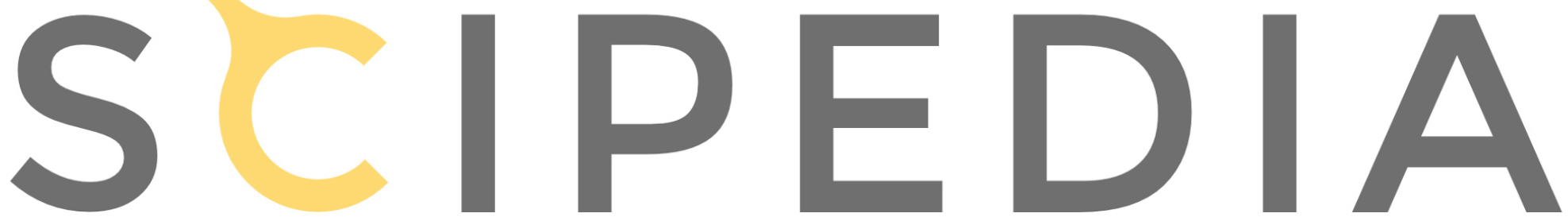

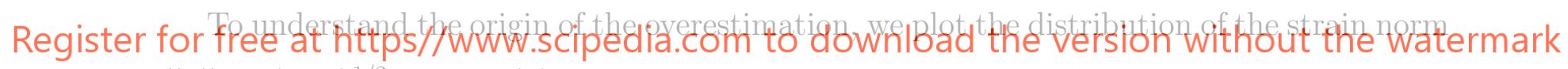
$\|\varepsilon\|:=(\varepsilon: \varepsilon)^{1 / 2}$ in Fig. 3(b) together with the distribution of the through-thickness mechani-

cal displacement $u_{2}$ in the deformed configuration of the pyramid in Fig. 3(c). Sharp changes of the strain are observed, particularly near the pyramid corners in Fig. 3(b), resulting in a significant and localized flexoelectric effect. This is in contrast to the assumptions underlying the analytical estimates, where a 1-D homogeneous distribution of the strain gradient is assumed. Furthermore, Fig. 3(c) shows that the pyramid undergoes a bending deformation in addition to the compressive deformation. Therefore, another important source of discrepancy is the bending deformation mode of the pyramid, mobilizing the transversal flexoelectric components neglected in the analytical model. In this simulation, a fully flexible support of the pyramid is considered, which uniformly distributes tractions as shown in Fig. 1. We consider later rigidly supported pyramids. Thus, both strain gradient localization due to compression and bending-induced strain gradients result in a higher effective flexoelectric response as compared to the naive calculation, resulting in an overestimation of the flexoelectric coefficient. 


\section{SYSTEMATIC DEPENDENCE OF EFFECTIVE FLEXOELECTRIC COEF- FICIENT ON GEOMETRY AND BOUNDARY CONDITIONS}

These results suggest that simple estimations such as those leading to Eq. (10) are not reliable to compute the flexoelectric response of truncated pyramids. In principle, the overestimation indicated by the normalized flexoelectric constant $\mu^{\prime}$ should strongly depend on the configuration and boundary conditions of the pyramid. To support this hypothesis, we perform a set of simulations considering different area ratios $(R)$ and inclination angles $(\alpha)$ of the truncated pyramid. Figure 4 presents the results for different pyramid configurations. It is clear that the overestimation increases noticeably by decreasing the area ratio and/or the inclination angle of the pyramid. The deformation (inset) shows that the main source of the overestimation is the bending deformation of the pyramid. For high inclination angle and high area ratio pyramids, the response is dominated by the compressive deformation

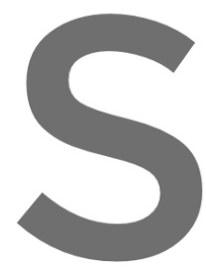
mode and the normalized flexoelectric constant approaches unity, i.e the overestimation
decreases. On the other hand, for loy inclination angles and low ared ratios, the pyranid
approaches a plate configuration and the response is mainly due to the bending deformation
mode. In this situation, the assumption of pure compressive deformation in Eq (8) is not

valid, leading to a noticeable overestimation of the flexoelectric constant (up to $\mu^{\prime}=45$ for Register for free at https//www.scipedia.com to download the version without the watermark $R=2.2$ and $\alpha=20^{\circ}$. This may partially explain the discrepancy between experimental

measurements using the pyramid compression method and theoretical estimates ${ }^{18}$.

The flexoelectric response of truncated pyramids also strongly depends on boundary conditions. To highlight this point, we perform additional simulations of the truncated pyramid by assuming a rigid bottom support, which constraints the displacements of the bottom face of the pyramid. In this situation, a non-uniform traction is induced on the bottom surface. We consider only the pyramids with an inclination angle of $\alpha=45^{\circ}$, which are the most common configurations ${ }^{11}$. The results in Fig. 4 show that the overestimation of the flexoelectric constant decreases for rigidly supported pyramids, where the bending deformation are precluded. However, we still observe an overestimation of the flexoelectric constant due to sharp changes of the strain field near the pyramid corners, as discussed earlier in Fig. 3(b). For intermediate conditions, where the bottom support is not fully rigid nor flexible, $\mu^{\prime}$ lies between the rigid and flexible results. By way of example, for the pyramid building unit in the experiments of Ref. ${ }^{3}$, we obtain $\mu^{\prime}=3.5$ in the flexibly 
